\title{
Software Solutions for ICME
}

\author{
G.J. SCHMITZ, ${ }^{1,9}$ A. ENGSTROM, ${ }^{2}$ R. BERNHARDT, ${ }^{3}$ U. PRAHL, ${ }^{4}$ \\ L. ADAM ${ }^{5}{ }^{J}$. SEYFARTH, ${ }^{5}$ M. APEL, ${ }^{1}$ C.AGELET DE SARACIBAR, ${ }^{6}$ \\ P. KORZHAVYI, ${ }^{7}$ J. ÅGREN, ${ }^{7}$ B. PATZAK ${ }^{8}$
}

1. Access e.V., Aachen, Germany. 2. Thermo-Calc Software AB, Stockholm, Sweden. 3. Simufact Engineering GmbH, Hamburg, Germany. 4. Steel Department of RWTH Aachen Univer-sity, Aachen, Germany. 5. e-xstream Engineering SARL, Luxembourg, Luxembourg. 6. Centre Internacional de Mètodes Numèrics a l'Enginyeria (CIMNE), Barcelona, Spain. 7. Royal Institute of Technology KTH Stockholm, Stockholm, Sweden. 8. Czech Technical University, Prague, Czech Republic. 9. e-mail: g.j.Schmitz@micress.de

\begin{abstract}
The Integrated Computational Materials Engineering expert group (ICMEg), a coordination activity of the European Commission, aims at developing a global and open standard for information exchange between the heterogeneous varieties of numerous simulation tools. The ICMEg consortium coordinates respective developments by a strategy of networking stakeholders in the first International Workshop on Software Solutions for ICME, compiling identified and relevant software tools into the Handbook of Software Solutions for ICME, discussing strategies for interoperability between different software tools during a second (planned) international workshop, and eventually proposing a scheme for standardized information exchange in a future book or document. The present article summarizes these respective actions to provide the ICME community with some additional insights and resources from which to help move this field forward.
\end{abstract}

\section{INTRODUCTION}

Integrated computational materials engineering (ICME) by its name and its nature draws on the combination and the simultaneous or consecutive use of a variety of software and modeling tools. This simple phrase immediately raises a number of questions, like the following: How does one combine tools? How does one select suitable tools? How does one decide on a specific tool? Eventually, before answering these questions, some even more direct issues arise, such as follows: Which tools are available at all? How does one become aware of suitable tools?

ICMEg, the Integrated Computational Materials Engineering expert group [1], a coordination activity of the European Commission, aims at developing a global open standard for information exchange between the heterogeneous varieties of numerous simulation tools. Once established, such an open and easily accessible formulation of a global standard will

- significantly facilitate the exchange of data between different tools;

- create new options and functionalities of the present tools;

- allow for easy integration between commercial and academic approaches and models;

- provide the pathway for life-cycle modeling of components/products;

- allow for global optimization of process chains instead of individually optimized models; and

- stimulate many further new developments, e.g., by including temperature and 
composition dependencies of data not being considered by now; by integration of data originating from electronic, atomistic, or mesoscopic models; and by stronger interaction with experimental data sets.

The ICMEg consortium [1] coordinates respective developments by a strategy of networking stake-holders in the first international workshop, compiling identified and relevant software tools into the Handbook of Software Solutions for ICME, discussing strategies for interoperability between different software tools during a second (planned) international workshop, and eventually proposing a scheme for standardized information exchange in a future book or document. The present article summarizes these respective actions to provide the ICME community with some additional insights and resources from which to help move this field forward.

\section{FIRST INTERNATIONAL WORKSHOP ON SOFTWARE SOLUTIONS FOR ICME}

Despite ICME currently emerging as a new and powerful discipline, coupling of different software tools is however still in its infancy and represents an issue consuming significant effort in terms of time and workforce, if a coupling is realized at all. The scope and goals of the first ICMEg workshop (see www.icmeg.info) were as follows (I) to convene and to network software providers/developers, both commercial and academic, interested in providing their solutions to the growing ICME community; (II) to create awareness about solutions and models currently available along all processing steps and across all scales; (III) to generate a comprehensive overview and a thematically structured inventory of such software solutions; (IV) to identify options emerging for individual codes by coupling them to other models/tools; (V) to identify needs for model functionalities upstream in the process chain, on the basis of requirements by models downstream in the value chain; and (VI) to discuss necessary steps to create a global and open standard for information exchange between different models/tools.

The workshop attracted more than 160 participants from 24 countries in 4 continents. About $40 \%$ of the attendees originated from academia. Many software companies had representation either in person or via different contributions, e.g., on bene- fits of standardization for software providers, thermodynamic databases for ICME, and simulation of casting/forming processes. Also, the strong interest of the user industry became obvious by participation and/or contributions of several individuals from aerospace companies and power utilities, steel and aluminum producers, and others. All abstracts were collected in an abstract booklet [2]

\section{ASSESSING THE FIRST WORKSHOP’S GOALS AGAINST OUTCOMES}

In the following discussion, the outcome of the workshop is assessed by comparing it with its scope and goals (in italics):

To convene and to network software providers/developers, both commercial and academic, interested in providing their solutions to the growing ICME community

In the end, more than 160 people attended the workshop. Sponsoring support from the software providers also clearly indicated the strong interest of the computational tool developers in ICME. Most participants provided extremely positive feedback in terms of having, e.g., made new business contacts and in terms of interesting discussions with scientists from other areas. This indicates the utility of the networking and knowledge transfer 
at this workshop, which is further supported by the participants' desire to attend the next meeting.

To create awareness about solutions and models currently available along all processing steps and across all scales

During the workshop, many simulation tools were presented and an even bigger number was at least briefly mentioned throughout the various talks presented. Additionally, several models/tools mentioned in the presentations were unknown to many participants. Actually the Handbook of Software Solutions for ICME, which is now being edited within the ICMEg project (see later discussion) summarizes the major available solutions and provides a thematically structured overview.

To identify needs for model functionalities upstream in the process chain, on the basis of requirements by models downstream in the value chain

Most "downstream" modeling tools suffer from a lack of specification of suitable initial conditions for simulations. These can most probably be provided (in the future) by simulation models in the "upstream”, portion of the process chain. The workshop emphasized this idea and triggered respective activities.

To discuss necessary steps to create a global and open standard for information exchange between different models/tools

As one of the first and most important steps toward standardization, an important question was raised during the final panel discussion. It referred to the willingness of the attending software companies/software providers to implement a communication standard. About 25 software tool representatives (both academic and commercial) were identified, and almost all (>90\%) were positive about implementing a future standard once it is defined. Based on this feedback, participants were asked for some actions to continue the mutual discussions and, thus, to foster and extend a new modelers network and to work toward the formulation of the standard: (i) to use and contribute to the forum on ICME available on the website www. icmeg.eu and (ii) to fill out the questionnaires on "Market Survey" [3] and "Modelling Tools.'’ [4].

To identify options emerging for individual codes by coupling them to other models/tools

These options should be mutually identified by the participating scientists and engineers developing/operating the individual codes and models, and there were many respective discussions.

\section{CONCLUSION FROM THE FIRST WORKSHOP}

In addition to the evaluation of the workshop's goals, an overview of many of the conclusions extracted from the ICMEg talks and interactive discussions is condensed here.

\section{Thermodynamics}

There is clearly a strong interest and willingness to couple different software tools, as well as a need to facilitate such coupling. A major issue is the need for uncertainty estimates on 
thermodynamics, as well as phase-based property data in general. It was mentioned that some work addressing the former issue had recently been initiated at the U.S. National Institute of Standards and Technology (NIST). A related well-known issue is that different phase names are used depending on the community and that there is a need for unique identifiers. It was proposed during the workshop that the space group together with Wyckoff positions will provide a unique phase identifier that could be used by various software tools internally, and this seems at the moment as a feasible solution. To avoid problems related to the use of different definitions for thermodynamic quantities, it was suggested that the definitions provided in the International Union of Pure and Applied Chemistry (IUPAC) Quantities, Units and Symbols in Physical Chemistry (Green Book) should be looked at and possibly recommended. With respect to standards, a thermodynamic "Exif" format that relates to input/output (I/O) of calculated thermodynamic data was discussed. However, it was further stated that due to CPU requirements, there will likely be a need for more than one coupling solution.

\section{Industrial Needs for ICME}

During the workshop, it turned out that simulation on the macroscale is widely accepted and in use in industry for a Technology Readiness Level (TRL, according to the European Commission definition) of 8-9. Microstructure evolution codes and even more detailed electronic, atomistic, and mesoscopic models by now have reached a TRL of approximately 4-5 or even less. Respective approaches are thus only used in specialized laboratories. Nevertheless, the benefit for microscale/mesoscale/mesoscopic simulations is widely accepted and the need for downscaling is clearly formulated from the industrial side. A more detailed summary of industrial needs for ICME is depicted in a separate publication in the present issue [5].

\section{Integration Platforms: Future Perspectives and Standardization}

There is a need to clearly define the user of the platform (designer, multi-domain expert, etc.) as this basically defines the requirements on the platform functionality. There is further a strong need to establish a common and standardized language (ontology) of the problem domain. This will help to identify and describe the entities in the model space as candidates for components.

The I/O-based approach and component approaches were compared and discussed. The advantage of component-based approaches consists primarily in their ability to support naturally multiple data formats by representing data as components, as well as their natural ability to support distributed resources. The component interfaces should be defined strictly as problem independent. Individual components representing exchanged data (such as fields and properties) should have attached metadata about physical meaning, units, etc. The end user should primarily be a multi-domain expert capable of defining a workflow in a workflow editor or by using a platform language. The process should result in a recommendation that contains the definitions of standardized components and their interfaces.

\section{Microstructure Models}

In view of a future common language for ICME, a widely accepted definition of "microstructures as the carrier of material properties" was given. It was stated that there is no process-property correlation. Instead, two correlations, process-microstructure and microstructure-properties, must always be considered. A proper treatment of microstructure evolution thus is inevitable and a key issue in ICME settings. A microstructure definition as " all internal structural features that affect material properties" was given, and it was stated that these features are "not confined to a mesoscopic or microscopic level." A compact and 
comprehensive overview about numerous models on the length scale of the microstructure was given in the plenary talk and further refined in the topical and cross-topical sessions. Different needs and options in the area of microstructure simulations were discussed. A specific example is the opportunity of storing simulated microstructures in digital microstructure database- $s$ even together with experimental data-and making them retrievable. Other options to improve microstructure models refer to the use of data being calculated by small-scale, discrete models associated with, for example, interfacial mobilities, anisotropic interfacial energies, thermomechanical properties of pure phases, and several others. On the scale of microstructure, especially for simulations of all solid-state processes, the specification of initial microstructures is demanding as the "incoming" material has already experienced a history that is "integrated" in the microstructure. Missing initial conditions for "downstream" simulations can in the future possibly be filled by microstructure databases comprising both experimental and/or simulated microstructures. "Ideal" microstructures being designed synthetically, socalled synthetic microstructures, may complement these databases and provide new insights.

In view of storing microstructure data and their standardized description, two major directions have been identified: (I) considering the microstructure as carrier of properties and thus using quantitative descriptions of the microstructure (in 2D and/or 3D) to handle the information or (II) remaining entirely on a level of microstructure models and just handle/store the model parameters. The first concept has the advantage that real and simulated microstructures can be treated in a transparent manner. However, severe problems in quantitatively describing the microstructures in an efficient manner and in extracting the relevant information may be encountered. The second concept represents a smart and lean solution but requires a standardized set of limited models and bears the risk of losing relevant microstructure information.

Along with defining a standard for data exchange-aiming to realize the "plug \& play" vision for ICME_-standards for benchmarks and error estimates (as are already being established in the thermodynamic and small-scale communities) are needed as well.

\section{Determination of Effective Properties}

Discussions about effective properties covered two principle questions: (I) How can we derive effective properties from microstructure information? (II) How can we transfer those effective properties to the component level? Those questions were dealt with in covering the topics of targeted properties, methods to derive effective values, and the issue of data transfer (I/O) for the means of setting up coupled analyses.

Effective material properties to be described from the microstructure need to cover a full range of different performances. Among those mentioned were mechanical, thermal, and electrical, as well as magnetic properties and permeability. Information of the individual effective response shall be generic and include means to describe both linear and nonlinear behavior, as well as stationary or un-stationary scenarios. The methods discussed to reach this goal were various. A principle differentiation was made between two different approaches. Those are numerical methods (finite element, fast Fourier transform, asymptotic, etc.) and analytics (mean field, bounding methods, etc.). During the discussions, it turned out that firstorder descriptions are the base, but also second-order descriptions might be needed and thus must be considered.

The input and output for those methods will have to include a clean header of metadata information describing the data. Such header information shall contain at least the general nature of the data, including a description via a generic nomenclature understood between different communities; trace- ability of the data, including from which measurement/method are the data originating; and additional and essential material information in addition to basic values, and how to apply them in terms of orientation, symmetry, etc. 
For data storage and exchange, a differentiation was made between essential and complementary data types. Those types can be stored either by means of pure tabular values of properties obtained or by means of a model that comes along with input parameters to compute those effective properties. Among the essential data were found the constitutive material properties, morphology (such as volume fraction), and boundary conditions. Complementary data types include, for example, information about interfacial properties and additional data concerning morphology (shape, statistical distributions, etc.). The needs for other enhancements of the data were also raised. Deterministic as well as statistical descriptions must be possible. Also, error indicators need to be included. An overall hierarchical structure of the data is required.

The transfer of data starts with a clearly defined assignment of input (microstructure information) and output (effective properties/model). This will have to include the issue of mapping over different "meshes" as well as the possibilities to cover re-meshing. Assignment to different entities of meshes must be covered (node, element, etc.). Finally, it is in general the coupling scheme that determines the nature of description of effective properties. During the workshop, the clear focus/need was set on coupling between the microscopic and the macroscopic level. Different principle approaches were thought of, including (I) weak coupling/linking, in which the larger scale is not aware of the microstructure; (II) strong coupling, in which effective properties are computed during runtime; and (III) partial coupling, where the macroscopic model is identified and depends on micro-level parameters.

In terms of the microstructure, effective proper- ties can include homogenized data (such as overall stiffness), but also localized quantities (e.g., stress distribution in the material to assess failure). Both types of data shall be accessible in the exchange scheme. Furthermore, the issue of self-consistency of models between the microscopic and macroscopic scales was discussed.

\section{Discrete Models}

The increasing importance of discrete modeling in materials research, especially since the advent of the Materials Genome Initiative (MGI) and ICME, calls for the development of standardized and efficient interfaces connecting the diverse models into integrated software tools. Standardized inter- faces between such tools and the end user are equally important.

At the end of electronic structure calculations, the first concern is always about the accuracy of density functional theory (DFT) functionals and other approximations involved, as well as about the numerical precision of the method employed to solve the Kohn-Sham equations. Standard molecular and solid-state tests have been developed by the scientific community and are routinely applied to test newly developed functionals. To test the precision of a DFT-based method, one can use, as a standard, a well-converged result obtained by using a highly precise method (e.g., by a full-potential all- electron technique). Numerical error in the course of high-throughput calculations can be automatically controlled (as, for example, it is done in the PyCMD error reduction tool). Standardization and automation are useful in practice of such research studies as they allow the researchers to delegate routine jobs to computers, make human tasks more intelligent, bring the analyses to a higher level, and reduce the risk of a human error.

However, these measures cannot fully guarantee the scientific correctness of the results obtained as there are always difficult cases that cannot be foreseen. However, there are some ways to misuse even a highly sophisticated scientific tool. To establish control over such cases, a quality assessment procedure (similar to the assessment procedure used in CALPHAD) has been proposed. To implement this, quality standards need to be established and maintained by the research community.

Storage of the results of ordinary or high-through- put studies in databases, with the 
purpose of delayed analysis or reuse, has been discussed as an important and resource-saving activity. Currently existing examples are user-specific databases in simulation packages, such as MedeA (Materials Design) and Materials Studio (BIOVIA/Accelrys), or public-access databases, such as the online library AFLOWLIB.ORG developed by the Quantum Espresso community. Formats and content of the stored data were discussed in several presentations by the academic and commercial software developers. The importance of storing the essential parameters of each computation, such as the details about the type and the number of basis functions, density of real-space and reciprocal space grids, exchange-correlation functional and other approximations used, and even the name of the research- er(s), was emphasized. In addition, one can use digital identifiers and checksums for checking the mutual compatibility of any two entries coming from the same or from two different database(s).

To be used in atomistic simulations, the information obtained on the level of electronic structure needs to be translated into interatomic interaction potentials (or force-fields). Achievements and challenges in deriving and using such potentials have been discussed. Taking into account that many different formalisms exist, and that there are certain degrees of freedom in definitions, introducing standards in this field may be very useful. Practical issues of storing the force-fields in databases (such as the ATLAS database or Inter- atomic Potential Repository at NIST) have been discussed. Standardization work needs to be per- formed to reach consensus on the units, definitions, terminology, assessments of accuracy/quality, and data formats.

General scientific issues such as how to keep the essential physical information upon coarse-graining or homogenization, or how to keep the accuracy when switching from one model of the same system to another, was a hot topic of discussion. Similar problems arise practically in all areas of modeling, ranging from the electronic structure to the mesoscopic levels. Standardization and automation are expected to have a positive influence on the following characteristics of discrete models used in materials simulations: scientific rigorousness and reliability, robustness (including reproducibility of modelling results), and compatibility.

\section{Process Modeling Software in ICME Settings}

Numerous discussions during the workshop and even after the event clearly show that ICME is an emerging field that attracts more and more interest, but there are still some uncertainties and obstacles to overcome. Currently, some of the main drivers for ICME developments include academia, government laboratories, and the aerospace and automotive industries. Maritime applications are in the early stages of using ICME. Overall, the approach is not yet widely spread across the entire supply chain. This is essentially caused by a lack of both human and financial resources. The supply chain is mostly dominated by small and midsized enterprise (SME) companies. Involving them in ICME and making them drivers for future developments will be the big challenge for ICME, but it will also provide a great opportunity.

Commercial software providers participating in the workshop discussed additional perspectives. Beyond the technical capabilities of the different simulation tools they identified four main aspects - costs, complexity, interface structure, and harmonization — which have to be considered as follows:

Costs

No software provider can provide all the numerous solutions (case dependent) needed to be combined to tackle complex ICME problems. The necessity of procuring and maintaining a number of software solutions automatically leads to cost issues with respect to licensing costs. Hence, new licensing and business models like pay per use or short-term subscriptions need to be considered. In the same context, hardware requirements were discussed. Computational efforts are and will be higher than for standard applications and thus require additional CPU 
capacity. Cloud solutions were discussed as one future alternative to enhance computational capacity temporarily.

\section{Complexity}

No individual user will be able to operate all the different tools and solutions in a proper way. ICME by its nature is thus and always will be a team effort. This fact could be a show stopper especially for the SME-based companies already mentioned. Hence, new approaches for joint services, consultancy, training, and education become an issue for both academia and for industrial users.

\section{Interface Structure}

It is clear that desired interface standards must be more than simple $\mathrm{I} / \mathrm{O}$ converters. The community needs to have smart and intelligent tools fulfilling certain criteria, such as follows: flexible format conversion considering widely spread engineering and calculation tools like MS Excel and data exchange via clouds; visualization (viewer, postprocessor); documentation and testing; search functions (machine readable); batch and queue functionality; adaptable, modular structures; workflow management; and traceability and parsing of data.

\section{Harmonization}

This point becomes essential to the success of ICME, and it should not be limited to a common nomenclature and taxonomy. It comprises also a common understanding and implementation of material models. Harmonization is important with respect to the parallelization of codes, or in simple words, adjustment of computation times plays a role especially in the industrial world. Last but not least, the community needs to harmonize data mining methods and databases for common use.

\section{Numerical Methods}

Different aspects related to the application of numerical methods in the computational simulation of multi-physics and multiscale problems were addressed as follows: (I) The importance of the consistency of the material models used all along the numerical simulation of a manufacturing process chain, to ensure a proper communication between the different models/software used, and the use of physical-based material models; (II) the efficiency of different numerical methods for data transfer between different meshes, either in re-meshing using a Lagrangian formulation or when using an Arbitrary Lagrangian-Eulerian (ALE) formulation; (III) general coupling strategies for multi-physics problems involving different space and time scales, e.g., fluid-structure interaction (FSI), fluid-structure-temperature interaction (FSTI), fluid-structure-contact interaction (FSCI), electromechanical-fluidstructure interaction (EMFSI), applying different independent codes that communicate using MPI within an HPC context; (IV) multiscale approaches for the numerical simulation of material forming processes involving a manufacturing chain; (V) a simulation platform, involving several software codes, to design new materials and systems within a multiscale framework, from atoms to structures, using discrete and continuum models; (VI) requirements and solutions for a complete process simulation; (VII) different numerical methods for the solution of engineering problems, such as finite difference method (FDM), finite element method (FEM), boundary element method (BEM), discrete element method (DEM), mesh-free method (MFM), particle finite element method (PFEM), extended finite element method (XFEM), isogeometric analysis (IGA) method, model order reduction (MOR), finite volume method (FVM), molecular dynamics (MD), representative volume element (RVE), and level set method (LSM). 


\section{First Workshop Summary}

From the presentations and discussions during the first workshop, the following overarching concluding remarks and gaps can also be pointed out:

- There is a lack of concreteness in the discussions. This is most likely linked to the intrinsic generic nature of ICME as a concept. To get more specific discussions in the future, sand-box scenarios for dedicated and well-specified problems of industrial relevance were proposed for the second workshop.

- The computational simulation of multi-physics and multiscale problems usually requires high- performance computing (HPC). This topic was not sufficiently addressed during the workshop, and it could be a more relevant topic for the next workshop.

- The validation and comparison of different soft- ware codes, solvers, methods, models, algorithms, and so on, in a series of simple, yet interesting, benchmarks for mapping and data transfer between different meshes, was missing. The participation of entities, such as National Agency for Finite Element Methods and Standards (NAFEMS), for instance, was also missing, and they could be invited to participate in the next workshop.

\section{HANDBOOK OF SOFTWARE SOLUTIONS FOR ICME}

As one of the outcomes of the first workshop, a Handbook of Software Solutions for ICME, which is being edited by G.J. Schmitz and U. Prahl, will be published by Wiley-VCH Weinheim. All workshop participants were strongly encouraged to contribute to this book, and the editors in the end recorded more than 70 volunteers from at least 12 countries eventually contributing as authors.

The Handbook is scheduled to appear at the time of the second international workshop in Barcelona, Spain, in April 2016.

\section{SECOND INTERNATIONAL WORKSHOP ON SOFTWARE SOLUTIONS FOR ICME}

The first workshop clearly emphasized the multiple benefits arising from combining models and tools. The workshop also got the different players in the different model worlds mutually acquainted.

The scope of the Second Workshop on Software Solutions for ICME [6] to be held in Barcelona in April 2016 will be on elaborating "How to combine different models and tools in practice"' and this scope can be summarized in one word: Interoperability.

This implicitly covers aspects like information exchange (standardized protocols), license issues, steering of multiple codes (platforms), examples of industrial applications, and many others.

The topics of this upcoming workshop will comprise but are not limited to (I) metadata, metadata schemata (e.g., metadata to describe microstructures, thermodynamics, properties, and models); (II) organizing and storing of data; (III) data structures for interoperability (e.g., filebased interoperability, HDF5 data structures, and MongoDB); (IV) work- flows, simulation platforms; (V) handling of datasets at different scales/hierarchical data structures; (VI) future roadmaps toward interoperability; and (VII) numerics and interoperability, which includes scenarios for interoperability among different mesh kinematic frameworks, re-meshing and mapping schemes, scenarios for interoperability between different mesh discretizations, scenarios for inter- operability between different types of numerical methods (e.g., FDM, FEM, BEM, DEM, MFM, and PFEM), and scenarios for parallel processing in multi-platforms. 
To account for the lessons learned from the first workshop, much more time will be devoted to discussions and the range of stakeholders will be slightly widened. To discuss " How to perform ICME in practice" and to have more specific examples in the generic ICME methodology, a number of sand-box scenarios of technological/industrial interest will be defined. In contrast to foundational engineering problems and test-case/simulation-benchmarks, it is however not intended to solve the respective problems. Focus is on the identification of tools being needed or being beneficial to tackle the respective topic and especially on the identification of the required information flow between these different tools and of a suitable metadata structure to describe the information. Such sandbox scenarios are foreseen, for example, in additive manufacturing technologies, plastic composites, a steel component, and an electronic assembly. Details about these scenarios are available on the ICMEg website. Some presentations during the first workshop evoked the impression that with respect to platforms and the "I'" in ICME, most issues seem to be almost solved. Most of these presentations, however, referred to integrated computational engineering (ICE) and did not really address the " $\mathrm{M}$ '. A conclusion is that focusing on any subsystem of ICME (e.g., ICE and CME) will defocus the real objective of ICME. Much work has already been done in any of the subsystems. The real ICME challenge is to get all these subsystems into one single, coherent structural framework.

\section{REFERENCES}

[1] G.J. Schmitz and U. Prahl, Integr. Mater. Manuf. Innov. 3, 2 (2014).

[2] ICMEg. 1st International Workshop on Software Solutions for Integrated Computational Materials Engineering. http:// web.access.rwth-aachen.de/MICRESS/ICMEg1/abstracts. html. Accessed 15 Oct 2015.

[3] ICMEg. ICMEg questionaire (Market Study Simulation Software). https://docs.google.com/forms/d/130ajVA_b5 tqUf9DCixhzX0tIg0Ieo6MWLU wDYMAAQ/view form?usp=send form. Accessed 15 Oct 2015.

[4] ICMEg. ICMEg questionaire (Survey on Modeling/Simulation Tools). https://docs.google.com/forms/d/186kvoljlL dUZ0tr4PANkJYbhau9tAQ67xnqTUX1P3M8/view form?usp=send form. Accessed 15 Oct 2015.

[5] A.W.A. Konter, H. Farivar, U. Prahl, and J. Post (in this issue).

[6] ICMEg. 2nd International Workshop on Software Solutions for Integrated Computational Materials Engineering. http:// congress.cimne.com/icme2016/frontal/default.asp. Accessed 19 October 2015. 